\title{
Soft X-Ray Imaging Design and Analysis Methods on DIII-D*)
}

\author{
Morgan W. SHAFER, Devon J. BATTAGLIA, Ezekial A. UNTERBERG, John M. CANIK, \\ Todd E. EVANS ${ }^{1)}$, Jeffrey H. HARRIS and Steven J. MEITNER \\ Oak Ridge National Laboratory, Oak Ridge, TN, USA \\ ${ }^{1)}$ General Atomics, San Diego, CA, USA
}

(Received 7 December 2010 / Accepted 11 February 2011)

\begin{abstract}
A new tangential 2D Soft X-Ray Imaging System (SXRIS) is being designed to examine the edge magnetic island structure in the lower X-point region of DIII-D. A synthetic diagnostic calculation coupled to 3D emissivity estimates is used to generate phantom images. Phillips-Tikhonov regularization is used to invert the phantom images for comparison to the original emissivity model. Noise level, island size, and equilibrium accuracy are scanned to assess the feasibility of detecting edge island structures. Models of typical DIII-D discharges indicate integration times $>1 \mathrm{~ms}$ with accurate equilibrium reconstruction are needed for small island $(<3 \mathrm{~cm})$ detection.
\end{abstract}

(c) 2011 The Japan Society of Plasma Science and Nuclear Fusion Research

Keywords: soft X-ray, imaging, ELMs, inversion, diagnostics, resonant magnetic perturbation

DOI: $10.1585 /$ pfr.6.2402041

\section{Introduction}

Control of edge localized modes (ELMs) is crucial for next step devices where the energy expelled can damage plasma-facing components [1]. Application of resonant magnetic perturbations (RMPs) has shown control of ELMs by changing the stability of the underlying edge equilibrium properties. Experiments on the DIII-D tokamak demonstrate full ELM suppression with this method [2]. Paradoxically, experiments with lithiumwall- conditioned plasmas and a different RMP mode spectrum on the NSTX spherical tokamak show the reverse effect: the application of the RMP triggers ELMs [3]. Furthermore, experiments on stellarator/heliotron devices (W7AS, Heliotron-J, and LHD) also show that resonant magnetic structures in the plasma edge play a role in ELM stability [4-6].

RMPs are theorized to induce overlapping island chains in the pedestal region that change the stability of ELMs by altering the density and/or temperature profiles through increased transport. This theory assumes the structure of RMP fields inside the plasma is similar to that in vacuum, neglecting a plasma response. In principle, however, the response of the (often rotating) plasma to externally imposed RMPs should shield (or amplify) the different RMP spatial harmonics [7]. Measurements of the actual island structure inside the plasma are thus needed to validate models for the effects of RMPs on ELM stability, especially for projections to future devices.

We use results and experience from tokamak and stellarator experiments to develop imaging/analysis techniques to detect structure induced by RMP fields inside the

author'se-mail: shafer@fusion.gat.com

*) This article is based on the presentation at the 20th International Toki Conference (ITC20). plasma. Soft X-ray (SXR) imaging can yield information on the magnetic topology of the edge plasma. Tangential viewing of SXR emission is routinely used on tokamaks and stellarators to detect core MHD modes [8]. Interpretation of the images is nontrivial due to the $3 \mathrm{D}$ chordal integration, and requires advanced inversion techniques [9]. The islands generated by RMPs generally have higher harmonics than those typically studied in the core and hence, are smaller. This motivates a study to assess how well inversions can be performed with this system.

This paper first addresses the design and a synthetic model used to estimate signal levels. Second, image inversion using Phillips-Tikhonov regularization [10] is assessed using a synthetic model where key parameters are scanned, e.g., noise level, island size, and equilibrium accuracy.

\section{DIII-D SXRIS Plan \& Synthetic Model}

The DIII-D Soft X-Ray Imaging System (SXRIS) relies on a tangential view with pinhole optics and an efficient CsI: Tl scintillator ( 40 photons per $1 \mathrm{keV}$ x-ray) that is coupled to a visible system consisting of a an $\mathrm{f} / 0.95$ imaging lens, coherent fiber bundle, and radiation-shielded CMOS camera. More details and an optical layout are found in Ref. [11]. The optical efficiency of this system was estimated to be approximately 0.1 electrons per $1 \mathrm{keV}$ $\mathrm{X}$-ray [11]. The efficiency limit is largely a result of the lens coupling of the image to the CMOS camera.

The tangential view is centered just above the lower $\mathrm{X}$-point region, which takes advantage of the poloidal field null and thereby expands the size of the magnetic structure otherwise viewed at the midplane. This view is illustrated in Fig. 1. The SXR emission originates from primar- 


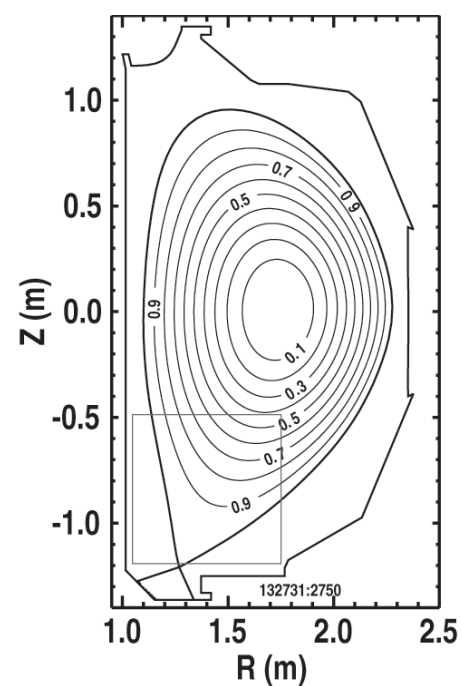

Fig. 1 DIII-D cross section with $\psi_{\mathrm{n}}$ contours. The box indicates tangential viewing area.

ily continuum radiation and higher- $Z$ impurity line radiation. SXR emission is chosen to avoid visible line radiation (a strong component in the edge and scrape-off layer (SOL)) and allow suitable signal at the top of a H-mode pedestal where $T_{\mathrm{e}} \geq 1 \mathrm{keV}$. A $7.5 \mu \mathrm{m}$ beryllium filter is used to discriminate against residual lower-temperature SXR emission in the $\psi_{\mathrm{n}}>0.98$ region, where $\psi_{\mathrm{n}}$ is the normalized poloidal flux.

A synthetic diagnostic model was developed to assess signal levels of tangential SXR imaging systems [12]. This model combines realistic camera geometry with a SXR emissivity source. The SXR spectrum is estimated with the CHIANTI database that takes $T_{\mathrm{e}}, n_{\mathrm{e}}$, and impurity concentrations as inputs [13]. This model was benchmarked against the SXR core-imaging camera on NSTX assuming axisymmetic emission and matched well to measured data [12].

For the work here, the synthetic diagnostic is coupled to a 3D SXR emission source. This source is generated using a single helicity island model $[14,15]$. This model generates a helical perturbation where the island width and phase are inputs for a given $m$ and $n$. A series of toroidal slices can be generated with the island perturbation, which is represented as a flat region in $\psi_{\mathrm{n}}$. 1D profiles of $T_{\mathrm{e}}$, $n_{\mathrm{e}}$, and $Z_{\text {eff }}$ are mapped to these slices based on $\psi_{\mathrm{n}}$. This creates the SXR emission to use in the synthetic model.

An example of the simulated SXR source is shown in Fig. 2 (a). A 3/1 island is located in the camera tangency plane at $\psi_{\mathrm{n}} \sim 0.9$ and is highlighted by a dotted line. As mentioned above, the island creates a flattening in the SXR emission. This effect is observed in data [15] and inherently assumes high parallel transport, such that temperature and density equilibrate along each field line. The flattening in the profile is a key component to the contrast observed in the diagnostic. In this case, the island width is $9 \mathrm{~cm}$ in the region of the camera (along the black line, be-

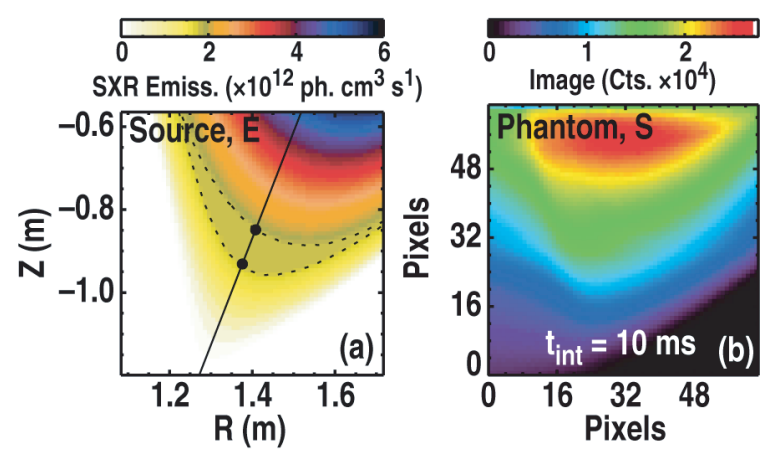

Fig. 2 Example SXR source emission (a) and phantom image (b).

tween solid black circles), while approximately $0.9 \mathrm{~cm}$ at the midplane. This helps illustrate the benefit of imaging the $\mathrm{X}$-point region.

Figure 2 (b) shows the camera "phantom" image. A $10 \mathrm{~ms}$ integration time is simulated. Currently, dark noise, read noise, and photon noise are simulated in the phantom image based on camera specifications [11]. At such long integration times, the image signal-to-noise ratio (SNR) is high (>100). This is important because an island perturbation on the mean emissivity is $1 \%-10 \%$ of the total signal. Therefore, the SNR of the perturbed image is $\sim 10$ [11]. Line integration obscures the flat region of emissivity created by the island. Image inversion discussed next is needed to extract meaningful data and determine the measurement range of the diagnostic.

\section{Image Inversion Assessment}

Image inversion is applied to phantom images generated by the above methods. Inversions are preferred in the case of RMP fields over other methods, such as frequency locking because RMP-induced islands should be static in the plasma. Phillips-Tikhonov regularization is used in this study, which approaches the inversion problem with a linear operation assuming smooth solutions. Regularization techniques take into account additional information and are commonly used over an iterative tomographic inversion when the problem is ill-posed [16,17], like it is here.

The imaging problem can be approximated by the equation $\mathbf{S}=\mathbf{L} \cdot \mathbf{E}+\mathbf{e}$, where $\mathbf{S}$ is the image, $\mathbf{E}$ is the emission source, $\mathbf{L}$ is the geometric transform, and $\mathbf{e}$ is the error between the measurement and the true source. The geometric transform maps "emission zones" from the source to detector pixels. A symmetry assumption is needed to generate a 3D solution from a $2 \mathrm{D}$ image. Here, the emission is assumed constant along the helical magnetic field lines. This assumption is sufficient for the phantom images, but may depend upon poloidally-asymmetric impurity transport during measurements. To construct $\mathbf{L}$, the location of sightlines corresponding to each detector pixel are mapped to a field line emission zone in 3D space corresponding to the source emission at the camera tangency 

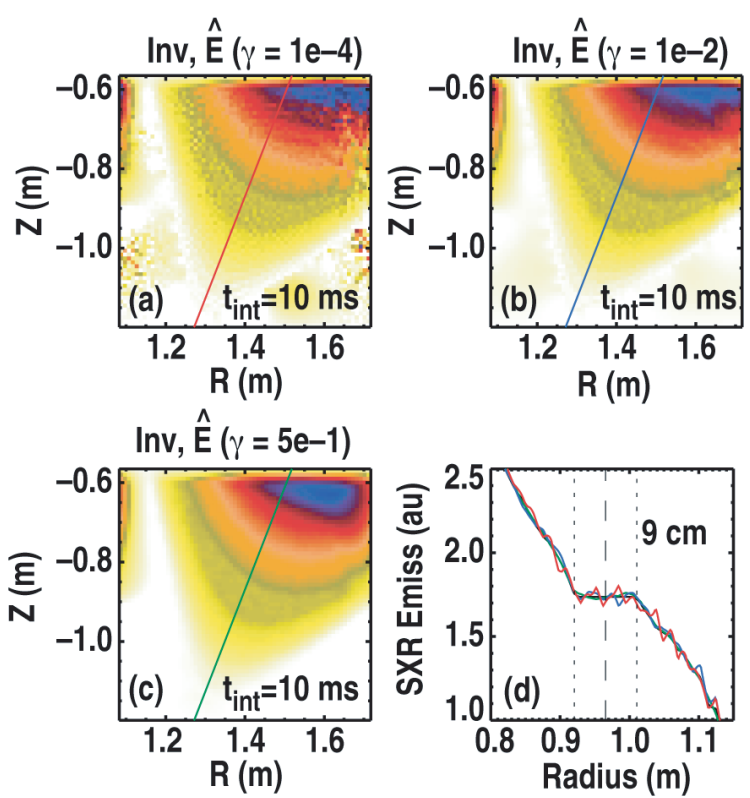

Fig. 3 Inverted images, $\gamma=10^{-4}(\mathrm{a}), \gamma=10^{-2}(\mathrm{~b}), \gamma=5 \times 10^{-1}$ (c), radial slices of inverted images through X-point (d).

view. Field line tracing is used to determine the emission zones.

The Phillips-Tikhonov method (described in $[10,18])$ minimizes the quantity, $\gamma|\mathbf{C} \cdot \mathbf{E}|^{2}+|\mathbf{S}-\mathbf{L} \cdot \mathbf{E}|^{2} / M$, which is composed of the error, $|\mathbf{S}-\mathbf{L} \cdot \mathbf{E}|^{2}$, and a cost function, $\gamma|\mathbf{C} \cdot \mathbf{E}|^{2}$. Here, $\mathbf{C}$ is the regularization operator, $\gamma$ is a weight for the operator (analogous to a Lagrange multiplier) and $M$ is the number of points in $\mathbf{S}$. The choice of $\mathbf{C}$ can be varied, but used here as the Laplacian operator, which assumes a diffusive nature to the smoothing. The construction of $\mathbf{C}$ is shown in Ref. [10]. The minimization procedure can be simplified to a linear operation, where the estimated source emission, $\hat{\mathbf{E}}(\gamma)$, is defined as $\hat{\mathbf{E}}(\gamma)=\left(\mathbf{L}^{T} \mathbf{L}+M \gamma \mathbf{C}^{T} \mathbf{C}\right)^{-1} \mathbf{L}^{T} \mathbf{S}$. The estimated source is now a function of $\gamma$, which determines how much of the cost function is applied, i.e. it is a trade-off between smoothness and noise. Iterative methods to find the optimal $\gamma$ exist $[10,18]$, but are not used here.

An example of the inversion of the phantom image from Fig. 2 (b) is shown in Fig. 3. Here, three values of $\gamma$ are used in parts $(\mathrm{a}-\mathrm{c}),\left(\gamma=10^{-4}, 10^{-2}, 5 \times 10^{-1}\right)$. This illustrates the relation between the smoothness and noise when compared to the source emission from Fig. 2 (a). Radial slices through the X-point are shown in Fig. 3 (d). The colors correspond to the line shown in parts $(\mathrm{a}-\mathrm{c})$. With low smoothing, edge distortions become more prominent, while central features remain similar to the source. All three weight levels show that the island is well reproduced when compared to the source (black). These are somewhat ideal conditions, with low noise levels, large islands, and well-matched equilibrium field lines. In reality, the performance of the diagnostic depends on these effects, which are quantified next.

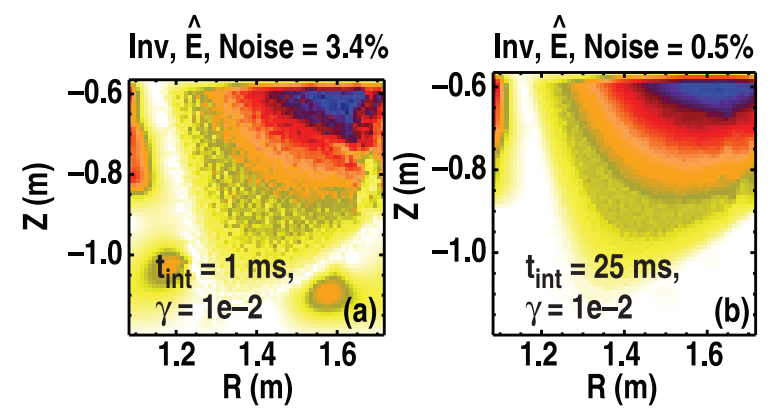

Fig. 4 Effect of noise in image inversion. 3.4\% noise, $1 \mathrm{~ms}$ integration (a) and $0.5 \%$ noise, $25 \mathrm{~ms}$ integration (b).

SXR Emissivity (au)
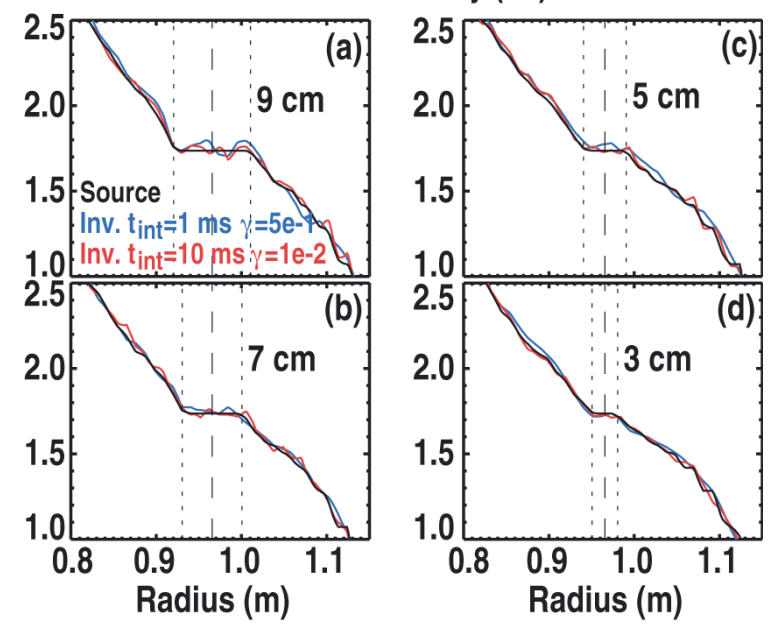

Fig. 5 Radial slices through the X-point for $3 / 1$ islands of $9 \mathrm{~cm}$

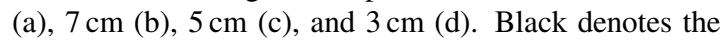
source emission, blue denotes $1 \mathrm{~ms}$ integration time, and red denotes $10 \mathrm{~ms}$ integration time.

First, the noise level is assessed. The noise level built into this model is controlled by the integration time. Both photon noise and dark noise are dependent on integration time, while the read noise acts as an offset. Two inverted images at different noise levels are shown in Fig. 4. The noise noted in the figure represent the flat-field noise as a percent of the mean image value. Noise levels of $3.4 \%$ and $0.5 \%$ are shown which correspond to $1 \mathrm{~ms}$ and $25 \mathrm{~ms}$ integration times. Unless signals levels are increased, this is a limit of the measurement $\left(t_{\text {int }}>1 \mathrm{~ms}\right)$.

Next, the simulated island width is varied from $9 \mathrm{~cm}$ to $3 \mathrm{~cm}$. Fig. 5 shows the profile cuts through the X-point for these different island sizes. The black traces in each represents the source emission, while the red and blue traces represent an inverted image. These results also couple in the effect of integration time and $\gamma$. The red traces in Fig. 5 represent a longer integration time $(10 \mathrm{~ms})$ and the blue traces represent the shorter integration time $(1 \mathrm{~ms})$. The red traces use a smaller $\gamma$, which smoothes the profiles less. For the shorter integration time, $\gamma$ was increased to counteract the effect of the noise shown in Fig. 4(a). An 

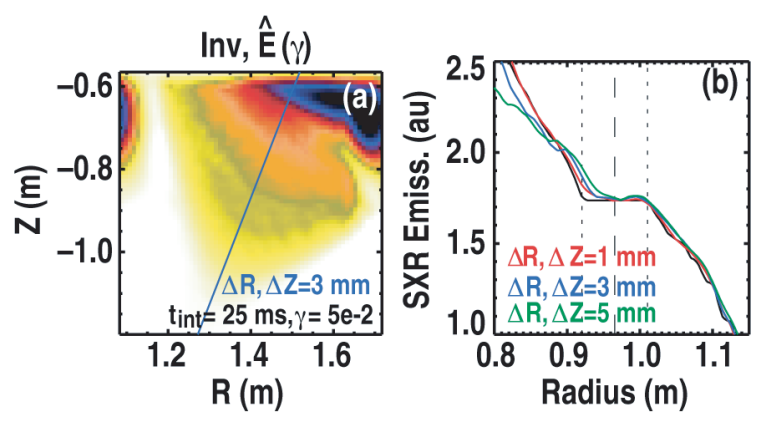

Fig. 6 Equilibrium shift effect on inverted images. Inverted image with $3 \mathrm{~mm}$ shift (a), profiles shifts for 1, 3, $5 \mathrm{~mm}$ (red, blue, green) (b).

increased $\gamma$ allows inversions for large islands $(\geq 5 \mathrm{~cm})$, while it smoothes over smaller islands. Longer integration times $(\geq 10 \mathrm{~ms})$ or signal enhancements are thus required for smaller islands $(<3 \mathrm{~cm})$.

Finally, the quality of the equilibrium field line tracing is assessed. The modeling thus far assumes the field lines traced to construct $\mathbf{L}$ matched exactly to the field structure used to construct the source emissivity. In reality, it is unlikely to match the field structure exactly, which requires accurate equilibrium reconstruction and image calibration. A simple test is used where the equilibrium field lines used in $\mathbf{L}$ are shifted in both $R$ and $Z$ by a small amount and the inverted images are compared. Figure 6 illustrates these results, where the field lines in $\mathbf{L}$ are shifted by 1,3 , and $5 \mathrm{~mm}$. An inverted image is shown in Fig. 6 (a) for shifts of $\Delta R, \Delta Z=3 \mathrm{~mm}$ with a long integration time $(25 \mathrm{~ms})$. This is needed to overcome the sensitivity of the field structure. Figure 6(b) compares three radial slices through X-point for different shifts. By increasing the shift, the inversion fails to reproduce the full island size modeled. The mismatch occurs only on the core-side of the island, which is not yet understood.

The three tests on the inversion method represent areas that will challenge the diagnostic performance. Long integration times and accurate field line modeling is needed to accurately invert island structure in the edge. The tests also depend upon the quality of the synthetic diagnostic modeling.
The work is ongoing with future tests planned. First, the inversions used were solely the Laplacian-based Phillips-Tikhonov regularization. We plan to test other cost functions that impose different constraints (e.g. maximum entropy or Bayesian statistics) [16,17]. Also, second-order optimization of the cost function to minimize error between pixels (where data is related) has been shown to enhance performance [18]. As mentioned above, the model matched well to data on NSTX, where the signal level has a strong dependence on the impurity concentration. For the cases modeled here, only carbon impurities were used. In future cases, inclusion of other high- $Z$ impurities, including neon or argon puffing, will be used to determine how much the signal levels are increased. These additional tests will likely add to the ability of this diagnostic. In summary, the DIII-D SXRIS will be poised to make definitive measurements regarding field penetration provided the conditions shown here are met.

The authors would like to acknowledge the useful discussions with Kevin Tritz and Satoshi Ohdachi. This work was supported by the US Department of Energy under DEAC05-00OR22725 and DE-FC02-04ER54698.

[1] H. Zohm, Plasma Phys. Control. Fusion 38, 105M (1996).

[2] T.E. Evans et al., Phys. Rev. Lett. 92, 235003 (2004).

[3] J.M. Canik et al., Phys. Rev. Lett. 104, 045001 (2010).

[4] F. Sano et al., Nucl. Fusion 45, 1557 (2005).

[5] S. Morita et al., Plasma Phys. Control. Fusion 48, A269 (2006).

[6] M. Hirsch et al., Plasma Phys. Control. Fusion 50, 053001 (2008).

[7] M. Becoulet et al., Nucl. Fusion 49, 085011 (2009).

[8] S. von Goeler et al., Rev. Sci. Instrum. 70, 599 (1999).

[9] S. Ohdachi, K. Toi, G. Fuchs, TEXTOR team and LHD experimental group, Plasma Sci. Technol. 8, 45 (2006).

[10] N. Iwama et al., Appl. Phys. Lett. 54, 502 (1989).

[11] M.W. Shafer et al., Rev. Sci. Instrum. 81, 10E534 (2010).

[12] D.J. Battaglia et al., Rev. Sci. Instrum. 81, 10E533 (2010).

[13] K.P. Dere, E. Landi, P.R. Young, G. Del Zanna, M. Landini and H.E. Mason, A\&A 498, 915 (2009).

[14] J.E. Menard et al., Nucl. Fusion 45, 539 (2005).

[15] S.P. Gerhardt et al., APS-DPP Meeting (2008), http:// meetings.aps.org/link/BAPS.2008.DPP.NP6.100

[16] M. Anton, Plasma Phys. Control. Fusion 38, 1849 (1996).

[17] L.C. Ingesson et al., Nucl. Fusion 38, 1675 (1997).

[18] S.E. Lee, Curr. Appl. Phys. 10, 893 (2010). 